\title{
Continuum many tent map inverse limits with homeomorphic postcritical $\omega$-limit sets
}

\author{
by \\ Chris Good (Birmingham) and Brian E. Raines (Waco, TX)
}

\begin{abstract}
We demonstrate that the set of topologically distinct inverse limit spaces of tent maps with a Cantor set for its postcritical $\omega$-limit set has cardinality of the continuum. The set of folding points (i.e. points at which the space is not homeomorphic to the product of a zero-dimensional set and an arc) of each of these spaces is also a Cantor set.
\end{abstract}

1. Introduction. The topological structure of inverse limits generated by unimodal maps has been studied extensively (cf. [2], [3], [9], [12], [14], $[15] \&[16])$, and one of the motivating conjectures is the following, due to W. T. Ingram: If $f$ and $g$ are tent map cores with $f \neq g$ then $\varliminf_{\lfloor}\{[0,1], f\}$ is

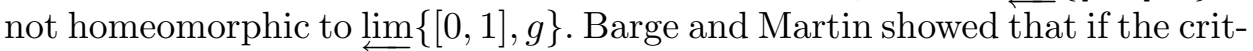
ical point $c$ of a unimodal map, $f$, is periodic or recurrent then $\lim _{\longleftarrow}\{[0,1], f\}$ will have endpoints [4]. Moreover, they showed that for $f$ a tent map core, if $c$ is periodic of period $n$ then $\lim _{\longleftarrow}\{[0,1], f\}$ has exactly $n$ endpoints. It follows that every other point has a neighborhood that is the product of a Cantor set and an arc. Recently Kailhofer has taken an important first step in proving Ingram's conjecture by showing that $\lim \{[0,1], f\}$ and $\lim \{[0,1], g\}$ are not homeomorphic when they have a periodic postcritical orbit and $f \neq g$ ([16] \& [17]).

At the other extreme is the set of tent maps with a dense postcritical orbit. Barge, Brucks and Diamond consider this case in [1]. Such a tent map $f$ generates a truly bizarre inverse limit space: $\lim \{[0,1], f\}$ has the property that every point is an endpoint and every neighborhood contains a homeomorph of every inverse limit obtainable by a tent map core. The set of parameters for which this occurs is large in both the topological and metric sense (cf. [8] \& [6]).

2000 Mathematics Subject Classification: 37B45, 37E05, 54F15, 54H20.

Key words and phrases: attractor, invariant set, inverse limits, unimodal, continuum, indecomposable. 
A generalization of the notion of endpoint in a continuum is that of a folding point. We show in [19] \& [18] that if $X$ is an indecomposable inverse limit of a map $f$ of an interval then every point in $X$ is either a folding point or it has a neighborhood homeomorphic to the product of a Cantor set and an arc. The collection of folding points of $X$, denoted by $\mathbb{F} \mathrm{d}(X)$, is preserved by any homeomorphism, so understanding the topological structure of this set and how it depends upon the dynamics of $f$ is a necessary step towards a proof of Ingram's conjecture. In [11] we use techniques from descriptive set theory to construct uncountably many (actually $\left.\omega_{1}\right)$ tent map cores $\left(f_{\gamma}\right)_{\gamma<\omega_{1}}$ with critical point $c_{\gamma}$ and with topologically distinct inverse limit spaces each with $\omega\left(c_{\gamma}\right)$ countable and $\mathbb{F} \mathrm{d}\left(\lim _{\longleftarrow}\left\{[0,1], f_{\gamma}\right\}\right)$ countable. These spaces are not homeomorphic because the sets of folding points are topologically distinct.

In this paper we examine the case that $f$ is a tent map core with nonrecurrent critical point $c$ and both $\omega(c)$ and $\mathbb{F} \mathrm{d}\left(\lim _{\{}\{[0,1], f\}\right)$ are Cantor sets. There is a dense set of parameters with cardinality of the continuum, $\mathfrak{c}$, in $(\sqrt{2}, 2]$ that generate such tent maps. Despite the fact that the collections of folding points are topologically identical and, in the subcase we consider, every proper subcontinuum is an arc, we show that there are continuum many non-homeomorphic inverse limit spaces generated by this type of tent map core.

2. Preliminaries. For completeness we include all of the relevant definitions. We encourage the reader unfamiliar with techniques from the theory of inverse limit spaces to consult [13] or [15].

By a continuum we mean a compact, connected metric space. We let $|A|$ stand for the cardinality of the set $A$. By cardinality of the continuum or size $\mathfrak{c}$ we mean the cardinality of the set $\mathbb{R}$, which is the same as $\mathcal{P}(\mathbb{N})$, the set of all subsets of $\mathbb{N}$. Occasionally we use the phrase "the continuum" to stand for the cardinal number $\mathfrak{c}=|\mathbb{R}|$.

If $A$ is a set then we say that $W$ is a word in $A$ provided $W$ is an ordered list of elements of $A$ with repetition allowed. We denote the concatenation of words $W_{1}$ and $W_{2}$ by simply $W_{1} W_{2}$, and by $W_{1}^{n}$ we mean the word $W_{1}$ concatenated with itself $n$ times.

Let $X$ be a compact topological space. Call a finite open cover, $\mathcal{U}=$ $\left\{U_{1}, \ldots, U_{n}\right\}$, of $X$ a chaining of $X$ or a chain provided $\bar{U}_{i} \cap \bar{U}_{j} \neq \emptyset$ if, and only if, $|i-j|<2$. We will call the elements of such a chain links. If $\operatorname{mesh}(\mathcal{U})<\varepsilon$ then we call $\mathcal{U}$ an $\varepsilon$-chain. If for every $\varepsilon>0$ there is an $\varepsilon$-chaining of $X$ then we say that $X$ is chainable. If $X$ is a chainable continuum and $x \in X$ then we say that $x$ is an endpoint of $X$ provided for every $A, B \subseteq X$ that are continua we have either $A \subseteq B$ or $B \subseteq A$.

Let $X$ be a chainable continuum. The following definition is due to Bruin [9]. Let $\mathcal{U}$ be a chaining of $X$. Let $\mathcal{L}=\left\{L_{1}, \ldots, L_{p}\right\}$ be a chain that 
refines $\mathcal{U}$. Let $L$ be a link of $\mathcal{U}$. We say that $\mathcal{L}$ turns in $L$ provided there is a link, $M$, in $\mathcal{U}$, adjacent to $L$, and integers $a$ and $b$ with $1 \leq a<b-1<b \leq p$ such that

(1) $L_{a}, L_{b} \subseteq M$

(2) $L_{j} \subseteq L \backslash M$ for some $a<j<b$,

(3) $\bigcup_{i=a}^{b} L_{i} \subseteq L \cup M$.

We call $L$ a turnlink. If it is true that every $\varepsilon$-chaining that refines $\mathcal{U}$ has a turnlink in $L$ then we call $L$ an essential turnlink.

Let $X$ be a continuum and $x \in X$. Call $x$ a folding point of $X$ if for every $\varepsilon>0$ there is an $\varepsilon$-chaining, $\mathcal{C}$, of $X$ that contains $x$ in an essential turnlink. Denote the set of folding points for a space $X$ by $\mathbb{F} \mathrm{d}(X)$.

Given a unimodal map, $h:[0,1] \rightarrow[0,1]$, with critical point $c_{h}$ we denote $\underset{\lim }{\longleftarrow}\{[0,1], h\}$ by $\mathbf{X}_{h}$ and the standard metric on $\mathbf{X}_{h}$ (induced by $|\cdot|$ on $[0,1]$ ) by $\widehat{d}_{h}$, and we call the set

$$
\operatorname{orb}\left(c_{h}\right)=\left\{h^{i}\left(c_{h}\right)\right\}_{i=0}^{\infty}
$$

the postcritical orbit of $h$. We say a map, $f$, is locally eventually onto, or l.e.o., provided for every $\varepsilon>0$ and for all $x \in[0,1]$ there is an integer $N$ so that $f^{N}[(x-\varepsilon, x+\varepsilon)]=[0,1]$. We have shown in [18] \& [19] that if $\widehat{x} \in \mathbf{X}_{h}$ then either $\widehat{x}$ has a neighborhood homeomorphic to a product of a Cantor set and an arc or it is a folding point. Moreover, we showed that if $h$ is l.e.o. then the collection $\mathbb{F} d\left(\mathbf{X}_{h}\right)$ consists of the points in $\mathbf{X}_{h}$ that always project into the $\omega$-limit set of $c_{h}$,

$$
\omega\left(c_{h}\right)=\bigcap_{n \in \mathbb{N}} \overline{\left\{h^{m}\left(c_{h}\right): m \geq n\right\}} .
$$

Given $q \in[1,2]$, we define the tent $\operatorname{map} T_{q}$ by

$$
T_{q}(x)= \begin{cases}q x & \text { if } x \leq 1 / 2, \\ q(1-x) & \text { if } x \geq 1 / 2 .\end{cases}
$$

We will restrict this map to its core, i.e. the interval $\left[T_{q}^{2}(1 / 2), T_{q}(1 / 2)\right]$, which is the only interval that contributes in a significant way to the inverse limit space, and we will rescale this restricted map, $\left.T_{q}\right|_{\left[T_{q}^{2}(1 / 2), T_{q}(1 / 2)\right]}$, to the entire interval. We call this rescaled map the tent map core and we denote it by $f_{q}:[0,1] \rightarrow[0,1]$. Notice that the critical point for $f_{q}$ is not $1 / 2$, rather it is the point $c=1-1 / q$. In order to ensure that $f_{q}$ is l.e.o. we also assume that $q \in[\sqrt{2}, 2]$. Due to renormalization of tent maps when $q \in[1, \sqrt{2}]$ this is not a restriction on the topology of the inverse limit space.

LEMMA 2.1. Let $h$ be the core of a tent map with critical point $c_{h}$ and $\omega\left(c_{h}\right)$ a Cantor set. Then $\mathbb{F} \mathrm{d}\left(\mathbf{X}_{h}\right)$ is also a Cantor set. 
Proof. A point $\widehat{x}$ is in $\mathbb{F} \mathrm{d}\left(\mathbf{X}_{h}\right)$ if and only if $\pi_{n}(\widehat{x})$ is an element of $\omega\left(c_{h}\right)$ for every $n \in \mathbb{N}$. Let $\widehat{x} \in \mathbb{F} \mathrm{d}\left(\mathbf{X}_{h}\right)$. Let $\varepsilon>0$ and choose $\delta>0$ and $N \in \mathbb{N}$ so that if $\widehat{z} \in \mathbf{X}_{h}$ and $\pi_{N}(\widehat{z})$ is within $\delta$ of $\pi_{N}(\widehat{x})$ then $d[\widehat{z}, \widehat{x}]<\varepsilon$. Since $\omega\left(c_{h}\right)$ is a Cantor set there is a point $z_{N} \in \omega\left(c_{h}\right)$ such that $\left|z_{N}-\pi_{N}(\widehat{x})\right|<\delta$. Since $h\left[\omega\left(c_{h}\right)\right]=\omega\left(c_{h}\right)$ it is easy to see that we can construct a point $\widehat{z} \in \mathbb{F} \mathrm{d}\left(\mathbf{X}_{h}\right)$ with $\pi_{N}(\widehat{z})=z_{N}$. Hence $\widehat{x}$ is not isolated in $\mathbb{F} \mathrm{d}\left(\mathbf{X}_{h}\right)$ and $\mathbb{F} \mathrm{d}\left(\mathbf{X}_{h}\right)$ is a Cantor set.

Let $i_{h}:[0,1] \rightarrow\{0,1, *\}$ be defined by

$$
i_{h}(x)= \begin{cases}0 & \text { if } x<c_{h}, \\ 1 & \text { if } x>c_{h}, \\ * & \text { if } x=c_{h} .\end{cases}
$$

For each point $x \in[0,1]$ define the itinerary of $x$ by

$$
I_{h}(x)=\left(i_{h}(x), i_{h} \circ h(x), i_{h} \circ h^{2}(x), \ldots\right)
$$

and, given an integer $M$, let the cylinder of diameter $M$ centered on $x$ be given by

$$
\left.I_{h}(x)\right|_{M}=\left(i_{h}(x), i_{h} \circ h(x), i_{h} \circ h^{2}(x), \ldots, i_{h} \circ h^{M}(x)\right) .
$$

The kneading sequence for $h$ is defined to be $K_{h}=I_{h}\left[h\left(c_{h}\right)\right]$.

The following results are well known and easy to prove [10]. Let $P_{h} \subseteq$ $[0,1]$ be the collection of precritical points for $h$, i.e. the collection of points that have $\mathrm{a} *$ in their itinerary. The following lemmas demonstrate that convergence in $[0,1]-P_{h}$ can be described completely using the itineraries.

Lemma 2.2. Let $h:[0,1] \rightarrow[0,1]$ be unimodal and l.e.o. and $\varepsilon>0$. Then there is an integer $N$ such that if $x, y \in[0,1] \backslash P_{h}$ and $\left.I_{h}(x)\right|_{N}=\left.I_{h}(y)\right|_{N}$ then $|x-y|<\varepsilon$.

Lemma 2.3. Let $h:[0,1] \rightarrow[0,1]$ be unimodal and l.e.o. and choose $N \in \mathbb{N}$. Then there is an $\varepsilon>0$ such that if $x, y \in[0,1] \backslash P_{h}$ and $|x-y|<\varepsilon$ then $\left.I_{h}(x)\right|_{N}=\left.I_{h}(y)\right|_{N}$.

As a result of these lemmas we see that we can identify points in the $\omega$-limit set of a point $y$ by simply analyzing the itinerary of $y$.

Theorem 2.4. Let $x, y \in[0,1]$. Then $x \in \omega(y)$ if and only if , for every $N \in \mathbb{N},\left.I_{h}(x)\right|_{N}$ occurs infinitely often in $I_{h}(y)$.

For each point $\widehat{x}=\left(x_{0}, x_{1}, \ldots\right)$ in $\mathbf{X}_{h}$ define the full itinerary for $\widehat{x}$ by

$$
\mathrm{Fi}_{h}(\widehat{x})=\left(\ldots, i_{h}\left(x_{3}\right), i_{h}\left(x_{2}\right), i_{h}\left(x_{1}\right) \cdot i_{h}\left(x_{0}\right), i_{h} \circ h\left(x_{0}\right), i_{h} \circ h^{2}\left(x_{0}\right), \ldots\right) .
$$

Notice that if $h$ is l.e.o. then $\mathrm{Fi}_{h}$ is a one-to-one map. Given a bi-infinite sequence $Z=\left(\ldots, \zeta_{-2}, \zeta_{-1} \cdot \zeta_{0}, \zeta_{1}, \zeta_{2}, \ldots\right)$ define the shift map by $\widehat{\sigma}(Z)=$ $\left(\ldots, \zeta_{-2}^{\prime}, \zeta_{-1}^{\prime} \cdot \zeta_{0}^{\prime}, \zeta_{1}^{\prime}, \zeta_{2}^{\prime}, \ldots\right)$ where $\zeta_{i}^{\prime}=\zeta_{i+1}$. Define the backwards itinerary 
for $\widehat{x}$ by

$$
\mathrm{Fi}_{h}^{-}(\widehat{x})=\left(\ldots, i_{h}\left(x_{3}\right), i_{h}\left(x_{2}\right), i_{h}\left(x_{1}\right)\right) .
$$

Let $X$ be a topological space and let $x \in X$. Then $K \subseteq X$ is called the composant containing $x$ provided $y \in K$ if and only if there is a proper subcontinuum of $X$ containing both $x$ and $y$. Brucks and Diamond [7] have shown that if $c_{h}$ is either periodic or non-recurrent then $\widehat{x}$ and $\widehat{y}$ are on the same composant of $\mathbf{X}_{h}$ if, and only if, the backwards itinerary of $\widehat{x}$ eventually agrees with the backwards itinerary of $\widehat{y}$. Define $\simeq$ on backwards itineraries by $\mathrm{Fi}_{h}^{-}(\widehat{x}) \simeq \mathrm{Fi}_{h}^{-}(\widehat{y})$ if there is an integer $n$ so that

$$
\left(\ldots, i_{h}\left(x_{n+1}\right), i_{h}\left(x_{n}\right)\right)=\left(\ldots, i_{h}\left(y_{n+1}\right), i_{h}\left(y_{n}\right)\right)
$$

Then Brucks and Diamond's result becomes: $\widehat{x}$ and $\widehat{y}$ are on the same composant if, and only if,

$$
\mathrm{Fi}_{h}^{-}(\widehat{x}) \simeq \mathrm{Fi}_{h}^{-}(\widehat{y}) .
$$

The maps we construct will have the property that $c_{h}$ is non-recurrent. Hence we can apply Brucks and Diamond's characterization of composants.

Given a point $\widehat{x} \in \mathbf{X}_{h}$ and an integer $M$ call the string

$$
\left.\operatorname{Fi}_{h}(\widehat{x})\right|_{-M, M}=\left(i_{h}\left(x_{M}\right), \ldots, i_{h}\left(x_{1}\right) \cdot i_{h}\left(x_{0}\right), i_{h} \circ h\left(x_{0}\right), \ldots, i_{h} \circ h^{M}\left(x_{0}\right)\right)
$$

the cylinder of diameter $M$ of $\operatorname{Fi}_{h}(\widehat{x})$.

The following lemmas are analogues of Lemmas $2.2 \& 2.3$ in the inverse limit space.

Lemma 2.5. Let $h:[0,1] \rightarrow[0,1]$ be l.e.o. and unimodal. Let $\varepsilon>0$. Then there is a positive integer $M$ with the property that if $\widehat{x}, \widehat{y} \in \mathbf{X}_{h}$ with $\left.\mathrm{Fi}_{h}(\widehat{x})\right|_{-M, M}=\left.\mathrm{Fi}_{h}(\widehat{y})\right|_{-M, M}$ and neither of $\mathrm{Fi}_{h}(\widehat{x})$ and $\mathrm{Fi}_{h}(\widehat{y})$ contains $*$ then $\widehat{d}_{h}[\widehat{x}, \widehat{y}]<\varepsilon$.

Proof. Let $\delta<\varepsilon / 4$. Since $h$ is l.e.o., there is an $M^{\prime}$ such that for any $x, y \in[0,1] \backslash P_{h}$ whenever $m \geq M^{\prime}$ and $\left.I_{h}(x)\right|_{M}=\left.I_{h}(y)\right|_{M}$ then $|x-y|<\delta$. Such an $M^{\prime}$ can be chosen by Lemma 2.2. Let $M \geq M^{\prime}$ be such that $1 / 2^{M}<$ $\varepsilon / 2$. Then suppose that $\widehat{x}, \widehat{y} \in \mathbf{X}_{h}$ with $\left.\operatorname{Fi}(\widehat{x})\right|_{-M, M}=\left.\operatorname{Fi}(\widehat{y})\right|_{-M, M}$. This implies that $\left|x_{j}-y_{j}\right|<\delta$ for all $j \leq M$. Hence we have the following simple calculation:

$$
\widehat{d}_{h}[\widehat{x}, \widehat{y}]=\sum_{i=0}^{M} \frac{\left|x_{i}-y_{i}\right|}{2^{i}}+\sum_{i>M} \frac{\left|x_{i}-y_{i}\right|}{2^{i}}<2 \delta+\frac{1}{2^{M}}<\varepsilon .
$$

Lemma 2.6. Let $h:[0,1] \rightarrow[0,1]$ be l.e.o. and unimodal. Let $M \in \mathbb{N}$. Then there is an $\varepsilon>0$ so that if $\widehat{x}, \widehat{y} \in \mathbf{X}_{h}$ with $\widehat{d}_{h}[\widehat{x}, \widehat{y}]<\varepsilon$ and neither $\mathrm{Fi}_{h}(\widehat{x})$ nor $\mathrm{Fi}_{h}(\widehat{y})$ contains $*$, then $\left.\mathrm{Fi}_{h}(\widehat{x})\right|_{-M, M}=\left.\mathrm{Fi}_{h}(\widehat{y})\right|_{-M, M}$.

Proof. Choose $\varepsilon^{\prime}>0$ so that if $x, y \in[0,1] \backslash P_{h}$ and $|x-y|<\varepsilon^{\prime}$ then $\left.I_{h}(x)\right|_{M}=\left.I_{h}(y)\right|_{M}$ (Lemma 2.3). Let $\varepsilon=\varepsilon^{\prime} / 2^{M}$, and let $\widehat{x}, \widehat{y} \in \mathbf{X}_{h}$ with 
$\widehat{d}_{h}(\widehat{x}, \widehat{y})<\varepsilon$. Clearly $\left|x_{M}-y_{M}\right| / 2^{M}<\varepsilon$; hence $\left|x_{M}-y_{M}\right|<2^{M} \varepsilon=\varepsilon^{\prime}$. Thus $\left.\mathrm{Fi}_{h}(\widehat{x})\right|_{-M, M}=\left.\mathrm{Fi}_{h}(\widehat{y})\right|_{-M, M}$.

Let ${ }^{\mathbb{N}} 2$ be the set of all functions from $\mathbb{N}$ to $\{0,1\}$ and given $\gamma \in{ }^{\mathbb{N}} 2$ denote the sequence $(\gamma(0), \gamma(1), \gamma(2), \ldots)$ by $\langle\gamma\rangle$ and the backwards sequence $(\ldots, \gamma(2), \gamma(1))$ by $\langle\gamma\rangle^{-}$. Let ${ }^{n} 2$ be the set of all functions from the set $\{0,1, \ldots, n-1\}$ to $\{0,1\}$ and let $<\mathbb{N}_{2}$ be $\bigcup_{n \in \mathbb{N}}{ }^{n} 2$. If $\gamma, \delta \in \mathbb{N}^{2}$ and there is an $N \in \mathbb{N}$ such that

$$
(\ldots, \gamma(N+2), \gamma(N+1), \gamma(N))=(\ldots, \delta(N+2), \delta(N+1), \delta(N))
$$

then $\langle\gamma\rangle^{-} \simeq\langle\delta\rangle^{-}$.

A sequence, $M$, in symbols 0 and 1 is primary provided it is not a $*-$ product, i.e. there is no finite word $W$ and sequence $\left(u_{i}\right)_{i \in \mathbb{N}}$ of symbols from $\{0,1\}$ with $M=W u_{1} W u_{2} W u_{3} \ldots$, i.e. $M$ is not the word $W$ followed by the symbol $u_{1}$, then $W$ followed by $u_{2}$, then $W$ followed by $u_{3}$, etc. The shift map, $\sigma$, on sequences is defined by $\sigma\left[\left(t_{0}, t_{1}, \ldots\right)\right]=\left(t_{1}, t_{2}, \ldots\right)$. We order sequences using the parity-lexicographic ordering, $\prec$. To define this order we first define $0<*<1$. Let $t=\left(t_{0}, t_{1}, t_{2}, \ldots\right)$ and $s=\left(s_{0}, s_{1}, s_{2}, \ldots\right)$ be sequences of zeroes and ones. Let $n$ be the least $j$ such that $t_{j} \neq s_{j}$. Let $m$ be the number of occurrences of the symbol 1 in the string $\left(t_{0}, t_{1}, \ldots, t_{n-1}\right)=$ $\left(s_{0}, s_{1}, \ldots, s_{n-1}\right)$. If $m$ is even then define $t \prec s$ if, and only if, $t_{m}<s_{m}$. If $m$ is odd then define $t \prec s$ if, and only if, $t_{m}>s_{m}$. It is easy to show that if $x<y$ then $I_{f}(x) \prec I_{f}(y)$. A sequence, $K$, is shift-maximal provided that for all $j \in \mathbb{N}, \sigma^{j}(K) \prec K$ or $\sigma^{j}(K)=K$.

The following theorem allows us to construct an infinite sequence of $0 \mathrm{~s}$ and 1 s that is the kneading sequence for a tent map core.

Theorem 2.7 ([10, Lemma III.1.6]). Let $K$ be a infinite sequence of $0 s$ and $1 s$ that is shift-maximal, primary and has $101^{\infty} \preceq K$. Then there is a parameter, $q$, in $[\sqrt{2}, 2]$ generating a tent map core, $f_{q}$, with kneading sequence $K$.

3. Inverse limit spaces with a Cantor set of folding points. We now construct uncountably many tent map cores which have non-homeomorphic inverse limit spaces with homeomorphic postcritical $\omega$-limit sets and homeomorphic collections of folding points in the inverse limit space. In fact the collection we construct has cardinality of the continuum $\mathfrak{c}$. We achieve this by constructing a distinct tent map inverse limit for each subset $J$ of $\mathbb{N}$ (in fact, for technical reasons, for each subset $J$ of $\mathbb{N}-\{1,2,3\}$ ). Given $J \subseteq \mathbb{N}$ we build a map $g_{J}$ with critical point $c_{J}$ such that $\omega\left(c_{J}\right)$ and $\mathbb{F} d\left(\mathbf{X}_{g_{J}}\right)$ are both Cantor sets with $\mathbb{F} d\left(\mathbf{X}_{g_{J}}\right)$ contained in uncountably many composants of $\mathbf{X}_{g_{J}}$. Moreover, $\mathbf{X}_{g_{J}}$ has the property that for each $j \in \mathbb{N}$ there is a composant $K$ of $\mathbf{X}_{g_{J}}$ with $j$ isolated points in $K \cap \mathbb{F} \mathrm{d}\left(\mathbf{X}_{g_{J}}\right)$ if, and only 
if, $j \in J$. This implies that if $J, J^{\prime} \subseteq \mathbb{N}$ with $J \neq J^{\prime}$ then $\mathbf{X}_{g_{J^{\prime}}}$ is not homeomorphic to $\mathbf{X}_{g_{J}}$.

Let $A=1001, B_{0}=1011101$ and $B_{1}=1010101$. For each $\gamma \in{ }^{<\mathbb{N}_{2}}$ let $B_{\langle\gamma\rangle}=B_{\gamma(0)} B_{\gamma(1)} \ldots B_{\gamma(m-1)}$ and $B_{\langle\gamma\rangle^{-}}=B_{\gamma(m-1)} \ldots B_{\gamma(2)} B_{\gamma(1)}$ where $m$ is the length of $\gamma$. For $\gamma \in{ }^{\mathbb{N}} 2$ we define $B_{\langle\gamma\rangle}$ and $B_{\langle\gamma\rangle^{-}}$analogously. Since $<\mathbb{N}_{2}$ is countable, let $\left(\gamma_{i}\right)_{i \in \mathbb{N}}={ }^{<\mathbb{N}} 2$ be some enumeration of $<\mathbb{N}_{2}$. Define

$$
W=B_{\left\langle\gamma_{1}\right\rangle} B_{\left\langle\gamma_{2}\right\rangle} B_{\left\langle\gamma_{1}\right\rangle} B_{\left\langle\gamma_{2}\right\rangle} B_{\left\langle\gamma_{3}\right\rangle} \cdots
$$

where the subscripts of the $\gamma$ 's follow the pattern

$$
1,1,2,1,2,3,1,2,3,4,1,2,3,4,5, \ldots
$$

Then $W$ is an infinite word containing every $B_{\langle\gamma\rangle}$ infinitely often for every $\gamma \in{ }^{<N} 2$. Let $s=A A W$; then it is easy to see that $s$ is shift-maximal, primary and $101^{\infty} \preceq s$. Hence by Theorem 2.7 there is a tent map core with kneading sequence equal to $s$. Let us fix this map $f$ and denote its kneading sequence by $K_{f}$ and its critical point by $c_{f}$.

Notice that $c_{f}$ is not recurrent under $f$, so we can apply Brucks and Diamond's characterization of composants [7]. It is also easy to see that $\omega\left(c_{f}\right)$ is a Cantor set (by Theorem 2.4, it is uncountable and it is not dense since the itinerary of any point in $\omega\left(c_{f}\right)$ is a (shift of a) sequence of $B_{0}$ s and $B_{1} \mathrm{~s}$, so $\mathbb{F} \mathrm{d}\left(\mathbf{X}_{f}\right)$ is a Cantor set. Notice that for all $\gamma, \delta \in \mathbb{N}_{2}$ and $n \in \mathbb{N}$, there is a point $\widehat{z} \in \mathbb{F} \mathrm{d}\left(\mathbf{X}_{f}\right)$ such that $\mathrm{Fi}_{f}(\widehat{z})=\sigma^{n}\left[B_{\langle\gamma\rangle^{-}} . B_{\langle\delta\rangle}\right]$. Thus we see that the set $\mathcal{K}=\left\{K: K\right.$ is a composant of $\mathbf{X}_{f}$ and $\left.K \cap \mathbb{F} \mathrm{d}\left(\mathbf{X}_{f}\right)\right\}$ is uncountable, because each such composant corresponds to some $\gamma \in{ }^{\mathbb{N}} 2$, which is an uncountable collection.

Let $J \subseteq \mathbb{N}$ be such that if $j \in J$ then $j \geq 4$. Let $\left(\widehat{z}_{j}\right)_{j \in J} \subseteq \mathbb{F} \mathrm{d}\left(\mathbf{X}_{f}\right)$ be a sequence of points on distinct composants of $\mathbf{X}_{f}$ such that

$$
\mathrm{Fi}_{f}\left(\widehat{z}_{j}\right)=B_{\left\langle\zeta_{j}\right\rangle-} \cdot B_{\left\langle\zeta_{j}\right\rangle}
$$

for some sequence $\left(\zeta_{j}\right)_{j \in \mathbb{N}} \subset{ }^{\mathbb{N}} 2$, with

$$
B_{\left\langle\zeta_{j}\right\rangle}=B_{1}^{j} B_{0}^{N_{1}} B_{1}^{j} B_{0}^{N_{2}} \cdots
$$

where $\left\{N_{i}\right\}_{i \in \mathbb{N}}$ is an increasing sequence of integers.

Define $Z_{j, N}^{-}$and $Z_{j, N}^{+}$such that

$$
\left.\mathrm{Fi}_{f}\left(\widehat{z}_{j}\right)\right|_{-7 N, 7 N}=Z_{j, N}^{-} \cdot Z_{j, N}^{+}=\left.B_{\left\langle\zeta_{j}\right\rangle_{-}} \cdot B_{\left\langle\zeta_{j}\right\rangle}\right|_{-7 N, 7 N} .
$$

Then each word $Z_{j, N}^{-}$is a terminal segment of $B_{\left\langle\zeta_{j}\right\rangle-}$ of length $7 N$. Since our "building blocks", the words $B_{0}$ and $B_{1}$, have length 7 , this guarantees that $Z_{j, N}^{-}$begins with a word $B_{0}$ or $B_{1}$ and $Z_{j, N}^{+}$ends with a word $B_{0}$ or $B_{1}$ rather than a fragment of such a word. Similarly, we denote $\operatorname{Fi}_{f}\left(\widehat{z}_{j}\right)$ by simply $Z_{j}^{-} \cdot Z_{j}^{+}$. 
We will use the following lemma later in the paper to demonstrate that certain folding points are on different composants of our inverse limit space.

Lemma 3.1. Let $j, k \in J$ with $j \neq k$. Then $Z_{j}^{-} \not Z_{k}^{-}$. Moreover, if $V$ is a non-empty word in $\{0,1\}$ and $j \in J$ then $Z_{j}^{-} V \not Z_{j}^{-}$.

Proof. Let $j, k \in J$ with $j \neq k$. Notice that in $Z_{j}^{-}$there are then infinitely many strings of the form $B_{0} B_{1}^{j} B_{0}$, but in $Z_{k}^{-}$there are no such strings. Hence there is no shift of these words after which they are equal. So $Z_{j}^{-} \nsucceq Z_{k}^{-}$.

Notice that since $\left\{N_{i}\right\}_{i \in \mathbb{N}}$ is an increasing sequence of integers, $Z_{j}^{-}$is not backwards periodic or pre-periodic. So if $V$ is a non-empty word in $\{0,1\}$, then $Z_{j}^{-} V$ will not have a tail that equals a tail of $Z_{j}^{-}$. Thus $Z_{j}^{-} V \nsucceq Z_{j}^{-}$.

Let $K_{j} \in \mathcal{K}$ be the composant of $\widehat{z}_{j}$. Let $\mathcal{K}^{\prime}=\mathcal{K} \backslash\left\{K_{j}: j \in J\right\}$. Since $J$ is countable and $\mathcal{K}$ is uncountable, $\mathcal{K}^{\prime}$ is also uncountable. Let $\Sigma=$ $\left\{\gamma \in \mathbb{N}_{2}\right.$ : there is a composant $K \in \mathcal{K}^{\prime}$ such that $\widehat{x} \in K$ if and only if $\left.\mathrm{Fi}_{f}^{-}(\widehat{x}) \simeq B_{\langle\gamma\rangle^{-}}\right\}$. Let $\Sigma_{n}$ be the set of all $\xi \in{ }^{n} 2$ such that there is an element $\gamma$ in $\Sigma$ with $\xi(i)=\gamma(i)$ for all $0 \leq i \leq n-1$. We say that $\xi$ is an initial segment of $\gamma$. Let $\Sigma_{<\mathbb{N}}=\bigcup_{n \in \mathbb{N}} \Sigma_{n}$.

For each $j \in J$ and $0 \leq k \leq j-1$ let

$$
W_{j, k}=1^{2 j-k-1} 01^{k} \text {. }
$$

Let $\phi: \mathbb{N}-\{1\} \rightarrow J \times \mathbb{N} \times \mathbb{N} \times \Sigma_{<\mathbb{N}}$ be a one-to-one function that satisfies the following conditions:

(1) if $\phi(r)=(j, k, N, \gamma)$ then $0 \leq k \leq j-1$ and $j \leq N$;

(2) if $\phi(r)=(j, k, N, \gamma)$ then the length of $\langle\gamma\rangle$ is greater than or equal to $N$;

(3) for each $j \in J$ and $0 \leq k \leq j-1$ if $\phi(r)=\left(j, k, N_{r}, \gamma_{r}\right)$ and $\phi(s)=\left(j, k, N_{s}, \gamma_{s}\right)$ and $r<s$ then $N_{r}<N_{s}$ and the length of $\left\langle\gamma_{r}\right\rangle$ is less than the length of $\left\langle\gamma_{s}\right\rangle$;

(4) for each $j \in J, 0 \leq k \leq j-1$ and $N \geq j$ the set $\left\{\gamma \in \Sigma_{<\mathbb{N}}\right.$ : there is some $r \in \mathbb{N}$ with $\phi(r)=(j, k, N, \gamma)\}$ is dense in $\Sigma_{\mathbb{N}}$.

Let $r \in \mathbb{N}-\{1\}$ and suppose that $\phi(r)=(j, k, N, \zeta)$. Let

$$
Z_{r}^{ \pm}=Z_{j, N}^{ \pm}, \quad W_{r}=W_{j, k}^{N}, \quad B_{r}=B_{\langle\zeta\rangle}, \quad B_{r}^{-}=B_{\langle\zeta\rangle^{-}} .
$$

Now recall that $K_{f}=A A B_{\left\langle\gamma_{1}\right\rangle} B_{\left\langle\gamma_{2}\right\rangle} B_{\left\langle\gamma_{1}\right\rangle} B_{\left\langle\gamma_{2}\right\rangle} B_{\left\langle\gamma_{3}\right\rangle} \ldots$ We can inductively re-label $K_{f}$ so that $K_{f}=A A S_{2} B_{2} S_{3} B_{3} \ldots$ in such a way that $S_{m}$ is long enough to contain every finite string from $S_{2} B_{2} S_{3} \ldots S_{m-1} B_{m-1}$ that occurs infinitely often and $B_{m}=B_{\langle\zeta\rangle}$, where $\phi(m)=(j, k, N, \zeta)$. We omit the case of $B_{1}$ here because we have already defined $B_{1}$ and $B_{0}$ as our basic building words, where we use $B_{m}$ as a label for some string $B_{\langle\zeta\rangle}$ where $\phi(m)=(j, k, N, \zeta)$. 
Let

$$
t=A A S_{1} B_{2}^{-} Z_{2}^{-} W_{2} Z_{2}^{+} B_{2} S_{3} B_{3}^{-} Z_{3}^{-} W_{3} Z_{3}^{+} B_{3} S_{4} \ldots
$$

Notice that $t$ is primary, shift-maximal and $101^{\infty} \preceq t$. Hence by Theorem 2.7 there is a tent map core $g$ with $K_{g}=t$. Let $c_{g}$ be the critical point for $g$. Notice also that each $B_{r}, Z_{r}^{ \pm}$and $S_{r}$ is a finite sequence of $B_{0} \mathrm{~s}$ and $B_{1} \mathrm{~s}$ and contains no more than three consecutive 1s. Moreover each $W_{r}$ equals $W_{j, k}^{N}$ for some $4 \leq j \leq N$ and $k \leq j$, and contains at least four consecutive 1 s. It is also true that $t$ contains an occurrence of 00 .

In order to fully describe the points in the set of folding points of $\mathbf{X}_{g}$ we need to first describe the points in $\omega\left(c_{g}\right)$. The next two lemmas begin that description.

Lemma 3.2. There is an embedding $\Phi: \omega\left(c_{f}\right) \rightarrow \omega\left(c_{g}\right)$ induced by the itinerary maps $I_{f}$ and $I_{g}$.

Proof. Let $z \in \omega\left(c_{f}\right)$. By the choice of $S_{i}$ there is a point $z^{\prime} \in \omega\left(c_{g}\right)$ with $I_{f}(z)=I_{g}\left(z^{\prime}\right)$, so define $\Phi(z)=z^{\prime}$. Since $\omega(c)$ contains no pre-critical points, by Lemmas $2.2 \& 2.3$ convergence in $\omega(c)$ is determined by initial segments of itineraries of points, so $\Phi$ is an embedding.

Lemma 3.3. If $z \in \omega\left(c_{g}\right)$, then $I_{g}(z)$ ends either in an infinite sequence of complete $B_{0} s, B_{1} s$, or $W_{j, k}$ s or it ends in $1^{\infty}$.

Proof. By Theorem 2.4, every initial segment of $I_{g}(z)$ occurs infinitely often in $K_{g}$. Therefore there are two possibilities for the tail of $I_{g}(z)$. Either $I_{g}(z)$ ends in an infinite sequence of complete $B_{0} \mathrm{~s}, B_{1} \mathrm{~s}$, or $W_{j, k} \mathrm{~s}$ (the building blocks of $B_{r}, Z_{r}^{ \pm}, S_{r}$ and $W_{r}$ in $K_{g}$ ), or it ends in some sequence of $0 \mathrm{~s}$ and $1 \mathrm{~s}$ whose initial segments arise from the internal structure of the $B_{0} \mathrm{~s}$, $B_{1} \mathrm{~s}$, or $W_{j, k}$ s. Since $B_{0}$ and $B_{1}$ are of fixed length and the length of $W_{r}$ increases with $r$, the second possibility can only arise from the $W_{r} \mathrm{~s}$. But now, since $W_{r}=W_{j, k}^{N}$ for some $j, k$ and $N$, this second possibility can only occur because initial segments of (the tail of) $I_{g}(z)$ occur in $W_{j, k}^{N}$ for some increasing sequence of $j$, which implies that $I_{g}(z)$ ends in $1^{\infty}$.

Now we completely describe every point in $\omega\left(c_{g}\right)$ in terms of its itinerary. In light of Lemmas $2.2 \& 2.3$ this gives us a complete picture of the set $\omega\left(c_{g}\right)$.

Lemma 3.4. If $z \in \omega\left(c_{g}\right)$ then, for some $0 \leq l \leq 7, \sigma^{l}\left[I_{g}(z)\right]$ is precisely one of the following:

(1) $B_{\langle\gamma\rangle}$ for some $\gamma \in{ }^{\mathbb{N}} 2$;

(2) $1^{\infty}$

(3) $1^{m} 01^{\infty}$ for some $m \in \mathbb{N}$;

(4) $B_{1}^{N} 1^{\infty}$ for some $N \in \mathbb{N}$;

(5) $W_{j, k}^{\infty}$ for some $j \in J$ and $0 \leq k \leq j-1$;

(6) $Z_{j, N}^{-} W_{j, k}^{\infty}$ for some $j \in J, 0 \leq k \leq j-1$ and $N \in \mathbb{N}$; 
(7) $W_{j, k}^{N} Z_{j}^{+}$for some $j \in J, k \leq j-1$, and $N \in \mathbb{N}$;

(8) $1^{k} Z_{j}^{+}$for some $j \in J$ and $k \leq j-1$;

(9) $1^{m} 01^{k} Z_{j}^{+}$for some $j \in J, m<2 j-k-1$, and $k \leq j-1$;

(10) $1^{k} B_{1}^{\infty}$ for some $k \in \mathbb{N}$;

(11) $1^{m} 01^{k} B_{1}^{\infty}$ for some $k, m \in \mathbb{N}$;

(12) $1^{k} Z_{j, N}^{+} B_{\langle\gamma\rangle}$ for some $j \in J, k \leq j-1, j \leq N$ and $\gamma \in \Sigma$;

(13) $1^{m} 01^{k} Z_{j, N}^{+} B\langle\gamma\rangle$ for some $j \in J, m<2 j-k-1, k \leq j-1, j \leq N$ and $\gamma \in \Sigma$;

(14) $W_{j, k}^{m} Z_{j, N}^{+} B_{\langle\gamma\rangle}$ for some $j \in J, k \leq j-1,0 \leq m \leq N, j \leq N$ and $\gamma \in \Sigma$

(15) $Z_{j, m}^{-} W_{j, k}^{N} Z_{j, N}^{+} B_{\langle\gamma\rangle}$ for some $j \in J, 0 \leq k \leq j-1, j \leq N, m \leq N$ and $\gamma \in \Sigma$

(16) $B_{\langle\xi\rangle}-Z_{j, N}^{-} W_{j, k}^{N} Z_{j, N}^{+} B_{\langle\gamma\rangle}$ for some $j \in J, 0 \leq k \leq j-1, j \leq N$, $\xi \in \Sigma_{<\mathbb{N}}$ and $\gamma \in \Sigma$ with $\xi$ an initial segment of $\gamma$.

Proof. Recall first of all that $B_{0}$ and $B_{1}$ are sequences of length 7 .

We begin by showing that each of these possible itineraries is realized in $\omega\left(c_{g}\right)$. Since $S_{i}$ was chosen to contain every word in $S_{1} S_{2} \ldots S_{i-1}$ that occurs infinitely often in $K_{f}$, we see that if $z^{\prime} \in \omega\left(c_{f}\right)$ then there will be a point $z \in \omega\left(c_{g}\right)$ with $I_{g}(z)=I_{f}\left(z^{\prime}\right)$. Thus for every $\gamma \in{ }^{\mathbb{N}} 2$ there is a $z \in \omega\left(c_{g}\right)$ with $I_{g}(z)=B_{\langle\gamma\rangle}$.

Notice that for each positive integer $k$, the word $W_{k, k-1}=1^{2 k-t} 01^{k-1}$ occurs infinitely often in $K_{g}$. This implies that for each positive integer $m$, there is a point $z \in \omega\left(c_{g}\right)$ with $I_{g}(z)=1^{m} 01^{\infty}$. Since $\omega\left(c_{g}\right)$ is shift-invariant, there is another point $z \in \omega\left(c_{g}\right)$ such that $I_{g}(z)=1^{\infty}$. Also, $W_{j, k}$ is always preceded by $Z_{j, N}^{-}$with $N$ increasing at each occurrence. But recall that $Z_{j, N}$ always starts with $B_{1}^{j}$, so as $j \rightarrow \infty$ we see that there is a point $z \in \omega\left(c_{g}\right)$ with itinerary $B_{1}^{N} 1^{\infty}$.

Clearly there is a point in $\omega\left(c_{g}\right)$ with itinerary $W_{j, k}^{\infty}$ for all $j \in J$ and $k \leq j-1$. Each occurrence of $W_{j, k}^{N}$ in $K_{g}$ is preceded by $Z_{j, N}^{-}$. So for $j \in J$, $k \leq j-1$ and $N \in \mathbb{N}$ there is a point in $\omega\left(c_{g}\right)$ with itinerary $Z_{j, N}^{-} W_{j, k}^{\infty}$.

Also, each occurrence of $W_{j, k}^{N}$ is followed by $Z_{j, N}^{+}$where $j \in J, k \leq j-1$ and $N \geq j$. Fix $N \in \mathbb{N}$, and notice that for all $M \geq N$ the string $W_{j, k}^{N} Z_{j, M}^{+}$ occurs infinitely often in $K_{g}$ as a tail of $W_{j, k}^{M} Z_{j, M}^{+}$. Thus there is a point in $\omega\left(c_{g}\right)$ with itinerary $W_{j, k}^{N} Z_{j}^{+}$for $j \in J, k \leq j-1$ and $N \in \mathbb{N}$. Since $\omega\left(c_{g}\right)$ is $\sigma$-invariant we also see that there are points in $\omega\left(c_{g}\right)$ with itineraries of the form $1^{k} Z_{j}^{+}$and $1^{m} 01^{k} Z_{j}^{+}$for $j \in J, m<2 j-k-1$ and $k<j$.

Recall that the words $Z_{j}^{+}$all begin with $B_{1}^{j}$, and notice that regardless of the choice of $j, k$ and $N$, each $W_{j, k}^{N}$ ends with a string of no more than $k 1$ s. 
So letting $j$ get arbitrarily large we see that the string $1^{m} B_{1}^{\infty}$ occurs as an itinerary in $\omega\left(c_{g}\right)$. Notice that $W_{j, k}^{N}$ ends with $1^{m} 01^{k}$ with $m \leq 2 j-k-1$ and $k \leq j-1$. Again letting $j$ get arbitrarily large we see that there is a point in $\omega\left(c_{g}\right)$ with itinerary $1^{m} 01^{k} B_{1}^{\infty}$ for any $m, k \in \mathbb{N}$.

Similarly, each $W_{j, k}^{N}$ is followed by $Z_{j, N}^{+} B_{r}$ where $\phi(r)=\gamma, B_{r}=B_{\langle\gamma\rangle}$ for some $\gamma \in \Sigma_{<\mathbb{N}}$, and the length of $\langle\gamma\rangle$ is greater than $N$. By property (4) of $\phi$ for a fixed $j \in J, k \leq j$ and $N \geq j$, the collection of all possible $\gamma \in \Sigma_{<\mathbb{N}}$ associated with $j, k, N$ equals $\Sigma_{<\mathbb{N}}$. So for each $\gamma \in \Sigma$ there is a sequence $\left(\gamma_{n}\right)_{n \in \mathbb{N}} \subseteq \Sigma_{<\mathbb{N}}$ of initial segments of $\gamma$ such that the string $W_{j, k}^{N} Z_{j, N}^{+} B_{\left\langle\gamma_{n}\right\rangle}$ occurs in $K_{g}$. Thus there is a point $z \in \omega\left(c_{g}\right)$ with itinerary $W_{j, k}^{N} Z_{j, N}^{+} B_{\langle\gamma\rangle}$ for all $\gamma \in \Sigma$. Because $\omega\left(c_{g}\right)$ is shift-invariant and because $W_{j, k}^{N}$ ends with a string of adjacent $1 \mathrm{~s}$ we see that each of $1^{m} Z_{j, N}^{+} B_{\langle\gamma\rangle}, 1^{m} 01^{k} Z_{j, N}^{+} B_{\langle\gamma\rangle}$ and $W_{j, k}^{m} Z_{j, N}^{+} B_{\langle\gamma\rangle}$ occurs as itinerary of a point in $\omega\left(c_{g}\right)$.

Fix $j \in J$ and $k \leq j$. Then for each $N \geq j$, the string $Z_{j, N}^{-} W_{j, k}^{N} Z_{j, N}^{+}$ occurs infinitely often in $K_{g}$. Since $Z_{j, M}^{-}$has $Z_{j, N}^{-}$as a terminal segment for $N \leq M$, we can see that (fixing $N \geq j$ ) for all $M \in \mathbb{N}$ the string $Z_{j, N}^{-} W_{j, k}^{\bar{M}} Z_{j, M}^{+}$occurs infinitely often in $K_{g}$. But as $M \rightarrow \infty$, the length of $W_{j, k}^{M}$ also goes to infinity. This demonstrates that there is a point $z \in \omega\left(c_{g}\right)$ with itinerary $I_{g}(z)=Z_{j, N}^{-} W_{j, k}^{\infty}$, and also there is another point $z \in \omega\left(c_{g}\right)$ with itinerary $I_{g}(z)=W_{j, k}^{\infty}$. However, since the string $Z_{j, N}^{-} W_{j, k}^{N} Z_{j, N}^{+}$occurs infinitely often in $K_{g}$, by the choice of the map $\phi$, for each $\gamma \in \Sigma_{<\mathbb{N}}$, the string $B_{\langle\gamma\rangle-} Z_{j, N}^{-} W_{j, k}^{N} Z_{j, N}^{+} B_{\langle\gamma\rangle}$ occurs infinitely often in $K_{g}$. This implies there are points $z \in \omega\left(c_{g}\right)$ with itineraries of the form $Z_{j, m}^{-} W_{j, k}^{N} Z_{j, N}^{+} B_{\langle\gamma\rangle}$ for $m \leq N$ and $\gamma \in \Sigma$ and of the form $B_{\langle\xi\rangle-} Z_{j, N}^{-} W_{j, k}^{N} Z_{j, N}^{+} B_{\langle\gamma\rangle}$ for $\xi \in \Sigma_{<\mathbb{N}}$, $\gamma \in \Sigma$ and $\xi$ an initial segment of $\gamma$. Hence each of the itineraries listed in the statement of the lemma is realized as a point in $\omega\left(c_{g}\right)$.

We now show that each $z \in \omega\left(c_{g}\right)$ has an itinerary of this sort. Either $I_{g}(z)$ contains a string of more than three adjacent $1 \mathrm{~s}$ or it does not. If not then $I_{g}(z)$ must be a shift of a word made up entirely of $B_{0}$ and $B_{1}$ words. So suppose that $I_{g}(z)$ does contain more than three adjacent $1 \mathrm{~s}$.

By Lemma 3.3, either $I_{g}(z)$ ends in $1^{\infty}$ or it does not.

CASE 1. Suppose first that

$$
I_{g}(z)=V 1^{\infty}
$$

where $V$ is a word in $\{0,1\}$. Let $k$ be the length of the word $V$. Since $z \in$ $\omega\left(c_{g}\right),\left.I_{g}(z)\right|_{k+N}$ occurs infinitely often in $K_{g}$ for all $N \in \mathbb{N}$. Since $\left.I_{g}(z)\right|_{k+N}$ ends with a string of $N 1 \mathrm{~s}$, it must "overlap" some word $W_{j, k}=1^{2 j-k-1} 01^{k}$ infinitely often with $j \geq N$ or $k \geq N$.

SubCASE $1 \mathrm{~A}$. $V$ is the null string and $I_{g}(z)=1^{\infty}$. 
SubCASE 1B. Suppose that $V$ contains a string of more than three adjacent 1s. Then $V$ is not a word simply in $B_{0}$ and $B_{1}$. This implies that as we increase $N$ the cylinder $\left.I_{g}(z)\right|_{k+N}$ must overlap $W_{j, k}$ with increasing $k$, and $V$ is eventually a terminal segment of $1^{2 j-k-1} 0$. Thus, by considering $N \geq k$ we see that there is an integer $m$ such that $V=1^{m} 0$, and

$$
I_{g}(z)=1^{m} 01^{\infty} \text {. }
$$

Subcase 1C. Suppose that $V$ has length at least 1 but does not contain a string of more than three adjacent 1s. Then $V$ is the terminal segment of a word in $B_{0}$ and $B_{1}$. Recall that in $K_{g}, W_{j, k}^{M}$ is always preceded by $Z_{j, M}^{-}$ with $M \geq j$, and recall that $Z_{j}^{+}$always starts with $B_{1}^{j}$. So as $j \rightarrow \infty, V$ is a terminal segment of an infinite collection of $Z_{j, M}^{-}$. But each $Z_{j, M}^{-}=$ $B_{\left\langle\zeta_{j}\right\rangle-\mid-7 M}$, which all end with $B_{1}^{j}$. So there is an integer $N$ and $0 \leq l \leq 7$ such that

$$
\sigma^{l}\left[I_{g}(z)\right]=B_{1}^{N} 1^{\infty} .
$$

CASE 2. Suppose that

$$
I_{g}(z) \neq V 1^{\infty}
$$

for any word $V$ in $\{0,1\}$. Then either $I_{g}(z)$ ends with $W_{j, k}^{\infty}$ for some $j \in J$ and $k \leq j$ or it does not.

Subcase 2A. Suppose that for some fixed $j \in J$ and $k \leq j$,

$$
I_{g}(z)=V W_{j, k}^{\infty}
$$

for some word $V$ in $\{0,1\}$. If $V$ is the empty word then

$$
I_{g}(z)=W_{j, k}^{\infty} \text {. }
$$

Similarly if the length of $V$ is less than or equal to seven then

$$
\sigma^{l}\left[I_{g}(z)\right]=W_{j, k}^{\infty}
$$

where $l$ is the length of $V$.

So suppose that $V$ is non-empty and has length greater than seven. Then let $r$ be the length of $V$ and notice that for each $M \geq \max \{r, j\}$, $\left.I_{g}(z)\right|_{r+2 j M}=V W_{j, k}^{M}$, which must occur infinitely often in $K_{g}$. But each occurrence of $W_{j, k}^{M}$ in $K_{g}$ is preceded by $Z_{j, M}^{-}$. Since $M$ was chosen to be larger than $r$, we see that $V$ must be the terminal segment of $Z_{j, M}^{-}$for all

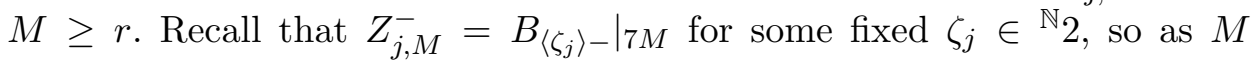
increases the terminal segment of $Z_{j, M}^{-}$is constant. Since $V$ starts with a fragment of $B_{0}$ or $B_{1}$, there is an integer $0 \leq l \leq 7$ such that

$$
\sigma^{l}\left[I_{g}(z)\right]=Z_{j, N}^{-} W_{j, k}^{\infty} .
$$

SubCASE 2B. Suppose that

$$
I_{g}(z) \neq V W_{j, k}^{\infty}
$$


for any $j \in J, k \leq j$ and word $V$ in $\{0,1\}$. By Lemma 3.3, $I_{g}(z)$ ends with a word of the form $B_{\langle\gamma\rangle}$ for some $\gamma \in{ }^{\mathbb{N}} 2$. So

$$
I_{g}(z)=V B_{\langle\gamma\rangle}
$$

with $V$ a word in $\{0,1\}$ containing more than three adjacent 1 s. Now either $V$ contains a 0 or it does not.

Subcase 2B(i). Suppose that there is no 0 in $V$. Then for some $3 \leq$ $m \in \mathbb{N}, I_{g}(z)=1^{m} B_{\langle\gamma\rangle}$. If $m \leq 7$, then $\sigma^{m}\left[I_{g}(z)\right]=B_{\langle\gamma\rangle}$ and we are done. So suppose that $m>7$. Then for every $N \geq m$, the string $\left.I_{g}(z)\right|_{m+N}=$ $\left.1^{m} B_{\langle\gamma\rangle}\right|_{m+N}$ must occur infinitely often in $K_{g}$. But the only places in $K_{g}$ that have a long string of $1 \mathrm{~s}$ followed by words $B_{0}$ and $B_{1}$ are of the form $W_{j, k}^{N} Z_{j, N}^{+} B_{\langle\delta\rangle}$ for some $j \in J, k \leq j \leq N$ and $\delta \in \Sigma_{\leq \mathbb{N}}$. The only word with more than seven adjacent $1 \mathrm{~s}$ in this expression is $W_{j, k}^{N}$. Thus either

$$
I_{g}(z)=1^{m} Z_{j, N}^{+} B_{\langle\delta\rangle}
$$

for $j \in J, m \leq j \leq N$ and $\delta \in \Sigma$, or

$$
I_{g}(z)=1^{m} Z_{j}^{+}
$$

for $m \in \mathbb{N}$ and $j \in J$, or

$$
I_{g}(z)=1^{m} B_{1}^{\infty}
$$

for $m \in \mathbb{N}$.

Subcase 2B(ii). Suppose that $V$ contains a 0 . Then, since $V$ contains more than three adjacent $1 \mathrm{~s}$, cylinders of $I_{g}(z)$ must overlap some word $W_{j, k}$ in $K_{g}$. But since $I_{g}(z)$ ends with $B_{\langle\gamma\rangle}$, it must be the case that there is a sequence of integers $\left(b_{i}\right)_{i \in \mathbb{N}}$ such that cylinders of $I_{g}(z)$ are contained in

$$
S_{b_{i}} B_{b_{i}}^{-} Z_{b_{i}}^{-} W_{b_{i}} Z_{b_{i}}^{+} B_{b_{i}}^{+} S_{b_{i+1}} \text {. }
$$

Let $k$ be the length of the last string of $1 \mathrm{~s}$ in $V$ that has length greater than 3. Let $m$ be the length of the string of $1 \mathrm{~s}$ in $V$ that precedes the string of length $k$. Then either we have a word $W_{m, k}$ contained in $I_{g}(z)$ or

$$
I_{g}(z)= \begin{cases}1^{m} 01^{k} Z_{j}^{+} & \text {where } j \in J, m<2 j-k-1, \\ & \text { and } 0 \leq k \leq j-1, \\ 1^{m} 01^{k} B_{1}^{\infty} & \text { where } m, k \in \mathbb{N}, \\ \left.1^{m} 01^{k} Z_{j, N}^{+} B_{\langle\gamma\rangle}\right\rangle & \text { for some } j \in J, m<2 j-k-1, \\ & 0 \leq k \leq j-1 \text { and } \gamma \in \Sigma .\end{cases}
$$

and we are done.

So suppose there is a word $W_{m, k}$ contained in $I_{g}(z)$. Let $N$ be such that $W_{m, k}^{N}$ occurs in $I_{g}(z)$. So $V$ is a terminal segment of $S_{b_{i}} B_{b_{i}}^{-} Z_{b_{i}}^{-} W_{b_{i}}$ with $\phi\left(b_{i}\right)=\left(m, k, N, \gamma_{i}\right)$ for some $\left(\gamma_{i}\right)_{i \in \mathbb{N}} \subseteq \Sigma_{<\mathbb{N}}$ with $N \geq M$. By property (5) of $\phi$ we see that as $i$ increases the length of each $\gamma_{i}$ also increases. This 
implies that $V$ is indeed a terminal segment of $B_{\left\langle\gamma_{i}\right\rangle}^{-} Z_{m, N}^{-} W_{m, k}^{N}$. Similarly, $\left.B_{\langle\gamma\rangle}\right|_{M}$ is an initial segment of some $Z_{m, N}^{+} B_{\left\langle\gamma_{i}\right\rangle}$. Together this means that there is an integer $l \leq 7$ such that

$$
\sigma^{l} I_{g}[z]= \begin{cases}W_{j, k}^{N} Z_{j}^{+} & \text {for some } j \in J, 0 \leq k \leq j-1 \\ & \text { and } N \in N, \\ W_{j, k}^{m} Z_{j, N}^{+} B_{\langle\gamma\rangle} & \text { for some } j \in J, k \leq j-1,0 \leq m \leq N, \\ & j \leq N \text { and } \gamma \in \Sigma, \\ Z_{j, m}^{-} W_{j, k}^{N} Z_{j, N}^{+} B_{\langle\gamma\rangle} & \text { for some } j \in J, 0 \leq k \leq j-1, \\ & j \leq N, m \leq N, \text { and } \gamma \in \Sigma, \\ B_{\langle\xi\rangle} Z_{j, N}^{-} W_{j, k}^{N} Z_{j, N}^{+} B_{\langle\gamma\rangle} & \text { for some } j \in J, 0 \leq k \leq j-1, j \leq N, \\ & \xi \in \Sigma_{<\mathbb{N}} \text { and } \gamma \in \Sigma \text { with } \xi \text { an initial } \\ & \text { segment of } \gamma,\end{cases}
$$

and we are done.

As an immediate application of the previous lemma we have:

Lemma 3.5. $\omega\left(c_{g}\right)$ is a Cantor set.

Proof. Since it is clear that $\omega\left(c_{g}\right)$ contains no intervals, it is enough to show that $\omega\left(c_{g}\right)$ contains no isolated points. Let $R$ be any finite word that occurs infinitely often in $K_{g}$, and let $U_{R}=\left\{x \in \omega\left(c_{g}\right): R\right.$ is an initial segment of $\left.I_{g}(x)\right\}$. We will show that $\omega\left(c_{g}\right)$ is a Cantor set by showing that $U_{R}$ is not a singleton. If $R$ occurs in $S_{i}$ (the words in the re-labeling of $K_{f}$ ) for infinitely many $i$, then $R$ occurs infinitely often in $K_{f}$. Since $\omega\left(c_{f}\right)$ is a Cantor set and since $\Phi$ is an embedding induced by $I_{f}$ there are no isolated points in $U_{R}$.

Suppose instead that $R$ does not occur infinitely often in $\bigcup_{i \in \mathbb{N}} S_{i}$. Then $R$ must contain more than three adjacent 1 s since these are the only words which did not already occur in $K_{f}$. Suppose first that $R=1^{m}$ or that $R=1^{m} 01^{k}$. Then for each $\gamma \in \Sigma$ there is a point in $U_{R}$ with itinerary $1^{m} 01^{k} Z_{j, n}^{+} B_{\langle\gamma\rangle}$. Thus $U_{R}$ is not a singleton.

Similarly, if $R=1^{k} Z_{j, N}^{+}$or $R=1^{m} 01^{k} Z_{j, N}^{+}$then for every $\gamma \in \Sigma$ there is a point in $U_{R}$ with itinerary $R B_{\langle\gamma\rangle}$. Hence $U_{R}$ is not a singleton.

Otherwise, $R$ contains a segment of the form $W_{j, k}^{N}$ for some $j \in J, 0 \leq$ $k \leq j-1$ and $N \in \mathbb{N}$. In $K_{g}$ such a segment is always followed by $Z_{j, M}^{+}$ for some integer $M \geq N$. For a given $j \in J, 0 \leq k \leq j-1$ and $M \in \mathbb{N}$, $B_{\langle\gamma\rangle}$ follows $W_{j, k}^{N} Z_{j, M}^{+}$for all $\gamma \in \Sigma_{<\mathbb{N}}$ with length longer than or equal to $M$ by property (4) in the definition of $\phi$. This implies that $U_{R}=\left\{x \in \omega\left(c_{g}\right)\right.$ : $R$ is an initial segment of $\left.I_{g}(x)\right\}$ is not a singleton.

The final case to consider is $R=B_{1}^{N} 1^{p}$. Then $R$ is an initial segment of a shift of $Z_{j, N}^{-} W_{j, k}^{m}$ for large enough $j \in J$. By the argument given in 
the previous paragraph we see that $U_{R}$ is not a singleton. Hence $\omega\left(c_{g}\right)$ is a Cantor set.

Hence $\omega\left(c_{f}\right)$ is homeomorphic to $\omega\left(c_{g}\right)$, by Lemma $2.1, \mathbb{F} d\left(\mathbf{X}_{f}\right)$ is homeomorphic to $\mathbb{F d}\left(\mathbf{X}_{g}\right)$ and all four sets are Cantor sets. But in order to arrive at the desired result we must know more about the possible full itineraries of all of the folding points. This will allow us later to use Brucks \& Diamond's characterization [7] to distinguish between the composants the various folding points are contained in.

Lemma 3.6. Let $\widehat{y} \in \mathbb{F} d\left(\mathbf{X}_{g}\right)$. Then for some integer $l \in \mathbb{Z}, \sigma^{l}\left[\mathrm{Fi}_{g}(\widehat{y})\right]$ is precisely one of the following:

(1) $B_{\langle\zeta\rangle-} . B_{\langle\gamma\rangle}$ for some $\zeta, \gamma \in \mathbb{N}_{2}$;

(2) $1^{-\infty} \cdot 1^{\infty}$

(3) $1^{-\infty} 0.1^{\infty}$

(4) $B_{1}^{-\infty} \cdot 1^{\infty}$;

(5) $W_{j, k}^{-\infty} \cdot W_{j, k}^{\infty}$ for some $j \in J$ and $0 \leq k \leq j-1$;

(6) $Z_{j}^{-} . W_{j, k}^{\infty}$ for some $j \in J$ and $0 \leq k \leq j-1$;

(7) $W_{j, k}^{-\infty} \cdot Z_{j}^{+}$for some $j \in J$ and $k \leq j-1$;

(8) $1^{-\infty} \cdot B_{1}^{\infty}$;

(9) $1^{-\infty} 01^{k} . B_{1}^{\infty}$ for some $k \in \mathbb{N}$;

(10) $B_{\langle\gamma\rangle-} Z_{j, N}^{-} W_{j, k}^{N} Z_{j, N}^{+} \cdot B_{\langle\gamma\rangle}$ for some $j \in J, 0 \leq k \leq j-1, j \leq N$, $\gamma \in \Sigma$.

Proof. This lemma follows from the fact that if $\widehat{y} \in \mathbb{F} \mathrm{d}\left(\mathbf{X}_{g}\right)$ then $\pi_{m}(\widehat{y}) \in$ $\omega\left(c_{g}\right)$ for every $m \in \mathbb{N}$, and from the characterization of itineraries of points in $\omega\left(c_{g}\right)$ (Lemma 3.4). It is clear that if $\widehat{y} \in \lim \{[0,1], g\}$ with $\operatorname{Fi}_{g}(\widehat{y})$ one of the bi-infinite words listed above then for each $n \in \mathbb{N}, \pi_{n}(\widehat{y}) \in \omega\left(c_{g}\right)$. So $\widehat{y} \in \mathbb{F} \mathrm{d}\left(\mathbf{X}_{g}\right)$.

Let $\widehat{y} \in \mathbb{F} \mathrm{d}\left(\mathbf{X}_{g}\right)$. We will show that the full itinerary of $\widehat{y}$ must be one of the bi-infinite words listed above. Every point in $\omega\left(c_{g}\right)$ has an itinerary that ends with one of:

(1) $1^{\infty}$

(2) $W_{j, k}^{\infty}$ for some $j \in J$ and $k \leq j-1$;

(3) $Z_{j}^{+}$for some $j \in J$;

(4) $B_{1}^{\infty}$;

(5) $B_{\langle\gamma\rangle}$ for some $\gamma \in \Sigma$, or

(6) $B_{\langle\gamma\rangle}$ for some $\gamma \in{ }^{\mathbb{N}} 2$ with $\gamma \notin \Sigma \cup\left\{1^{\infty}\right\}$.

Since this lemma addresses the structure of full itineraries up to a forward or backward shift we lose no generality in assuming that the itinerary of $\pi_{1}(\widehat{y})$ is one of the above six cases. 
Suppose that $\pi_{1}(\widehat{y})$ has itinerary $1^{\infty}$. Then either there is an integer $m>1$ such that $\pi_{m}(\widehat{y})$ has itinerary $01^{\infty}$ or there is no such $m$. If there is no such integer $m$ then

$$
\mathrm{Fi}_{g}(\widehat{y})=1^{-\infty} \cdot 1^{\infty}
$$

So suppose there is such an integer $m$. If for all $r>m$, the itinerary $I_{g}\left[\pi_{r}(\widehat{y})\right]$ equals $1^{k-m-1} 01^{\infty}$, then

$$
\sigma^{m-1}\left[\operatorname{Fi}_{g}(\widehat{y})\right]=1^{-\infty} 0.1^{\infty} .
$$

If that is not the case, then by Lemma 3.4 for each $r>m$ the itinerary $I_{g}\left[\pi_{7 r}(\widehat{y})\right]$ is $B_{1}^{K} 1^{\infty}$. Thus

$$
\sigma^{m-1}\left[\mathrm{Fi}_{g}(\widehat{y})\right]=B_{1}^{\infty} \cdot 1^{\infty}
$$

According to Lemma 3.4 these are the only possibilities that end in $1^{\infty}$.

Next, suppose that $I_{g}\left[\pi_{1}(\widehat{y})\right]=W_{j, k}^{\infty}$. If for every $m>1, \pi_{7 m}(\widehat{y})$ has itinerary $W_{j, k}^{\infty}$ then

$$
\mathrm{Fi}_{g}(\widehat{y})=W_{j, k}^{-\infty} \cdot W_{j, k}^{\infty} .
$$

The other possibility is that there is some integer $m>1$ such that $\pi_{7 m}(\widehat{y})$ has itinerary $Z_{j, N}^{-} W_{j, k}^{\infty}$ with $N \geq j$. Clearly then there is some $l \in \mathbb{Z}$ such that

$$
\sigma^{l}\left[\operatorname{Fi}_{g}(\widehat{y})\right]=Z_{j}^{-} \cdot W_{j, k}^{\infty} .
$$

Suppose now that the itinerary of $\pi_{1}(\widehat{y})$ is $Z_{j}^{+}$for some $j \in J$. It is certainly possible that

$$
\mathrm{Fi}_{g}(\widehat{y})=Z_{j}^{-} \cdot Z_{j}^{+}
$$

but since the $Z_{j}$ words were chosen from ${ }^{\mathbb{N}} 2$, this is a subcase of

$$
\mathrm{Fi}_{g}(\widehat{y})=B_{\langle\zeta\rangle-} \cdot B_{\langle\gamma\rangle} \cdot
$$

According to Lemma 3.4 the other possibility is that there is an integer $M>1$ such that $\pi_{7 m}(\widehat{y})$ has itinerary $W_{j, k}^{N} Z_{j}^{+}$for some $k \leq j-1$ and $N \in \mathbb{N}$, for all $m \geq M$. Thus

for some $l \in \mathbb{Z}$.

$$
\sigma^{l}\left[\operatorname{Fi}_{g}(\widehat{y})\right]=W_{j, k}^{-\infty} \cdot Z_{j}^{+}
$$

The next case to consider is $I_{g}\left[\pi_{1}(\widehat{y})\right]=B_{1}^{\infty}$. If for each $m>1$ there is a $\xi_{m} \in{ }^{<N} 2$ such that $I_{g}\left[\pi_{m}(\widehat{y})\right]=B_{\left\langle\xi_{m}\right\rangle-} B_{1}^{\infty}$ then

$$
\mathrm{Fi}_{g}(\widehat{y})=B_{\langle\xi\rangle-} B_{1 \infty}
$$

for some $\xi \in{ }^{\mathbb{N}} 2$ and, since $1^{\infty} \in{ }^{N} 2$, this possibility is covered. If this is not the case then there is an integer $M$ such that $I_{g}\left[\pi_{M}(\widehat{y})\right]=1^{k} B_{1}^{\infty}$ for some $k \in \mathbb{N}$. Now either for all $m \geq M, I_{g}\left[\pi_{m}(\widehat{y})\right]=1^{r} B_{1}^{\infty}$ for some $r \in \mathbb{N}$, or not. If so, then we have

$$
\sigma^{l}\left[\operatorname{Fi}_{g}(\widehat{y})\right]=1^{-\infty} . B_{1}^{\infty}
$$


for some integer $l$. If not, then according to Lemma 3.4, the only other possibility is that there is a $P \in \mathbb{N}$ such that for all $p \geq P$ there is an integer $m_{p} \in \mathbb{N}$ such that $I_{g}\left[\pi_{p}(\widehat{y})\right]=1^{m_{p}} 01^{k} B_{1}^{\infty}$ for some fixed $k \in \mathbb{N}$. Then for some $l \in \mathbb{Z}$,

$$
\sigma^{l}\left[\operatorname{Fi}_{g}(\widehat{y})\right]=1^{-\infty} 01^{k} \cdot B_{1}^{\infty}
$$

Finally, suppose that the itinerary of $\pi_{1}(\widehat{y})$ is $B_{\langle\gamma\rangle}$ for some $\gamma \in \Sigma$. Since $\Sigma \subseteq \mathbb{N}_{2}$ it could be the case that

$$
\mathrm{Fi}_{g}(\widehat{y})=B_{\langle\zeta\rangle-} \cdot B_{\langle\gamma\rangle}
$$

for some $\zeta \in{ }^{\mathbb{N}} 2$. If this is not the case then there is an integer $m>1$ such that the itinerary of $\pi_{7 m}(\widehat{y})$ has $W_{j, k}^{N}$ as an initial segment for some $j \in J$, $k \leq j-1$ and $j \leq N$. According to Lemma 3.4 there is an integer $M$ such that if $m \geq M$ then the itinerary of $\pi_{7 m}(\widehat{y})$ is $B_{\left\langle\xi_{m}\right\rangle-} Z_{j, N}^{-} W_{j, k}^{N} Z_{j, N}^{+} B_{\langle\gamma\rangle}$ for some $j \in J, k \leq j-1, j \leq N, \xi_{m} \in \Sigma_{<\mathbb{N}}$ and $\gamma \in \Sigma$, with $\xi_{m}$ an initial segment of $\gamma$. Thus

for some $l \in \mathbb{Z}$.

$$
\sigma^{l}\left[\mathrm{Fi}_{g}(\widehat{y})\right]=B_{\langle\gamma\rangle-} Z_{j, N}^{-} W_{j, k}^{N} Z_{j, N}^{+} \cdot B_{\langle\gamma\rangle}
$$

Now we examine the various composants containing folding points by using Brucks and Diamond's characterization [7].

Lemma 3.7. Let $K_{\gamma}$ be a composant of $\mathbf{X}_{g}$ such that $K_{\gamma}$ contains $\widehat{v} \in$ $\mathbb{F} \mathrm{d}\left(\mathbf{X}_{g}\right)$ where $\mathrm{Fi}_{g}(\widehat{v})=B_{\langle\gamma\rangle-} \cdot V$ for some $\gamma \in \Sigma-\left\{1^{\infty}\right\}$ and $V$ a word in $\{0,1\}$. Then $K_{\gamma} \cap \mathbb{F} \mathrm{d}\left(\mathbf{X}_{g}\right)$ is a Cantor set.

Proof. Let $\widehat{d}$ be a point in $\mathbb{F d}\left(\mathbf{X}_{g}\right) \cap K_{\gamma}$. Then there is a word $\zeta \in \Sigma$ such that $B_{\langle\zeta\rangle-} \simeq B_{\langle\gamma\rangle-}$ and $\mathrm{Fi}_{g}(\widehat{d})=B_{\langle\zeta\rangle-} V_{0} \cdot V_{1}$ for some words $V_{0}$ and $V_{1}$ in $\{0,1\}$ (so that $\pi_{1}(\widehat{d})=V_{1}$ ). Since $\sigma$ is a homeomorphism on $\mathbf{X}_{g}$, we lose no generality in only considering the case that $V_{0}$ is empty. Then either $V_{1}=B_{\langle\xi\rangle}$ for some $\xi \in{ }^{\mathbb{N}} 2$ (in which case $\widehat{d}$ is clearly not isolated in $K_{\gamma}$ ) or

$$
V_{1}=Z_{j, N}^{-} W_{j, k}^{N} Z_{j, N}^{+} B_{\langle\zeta\rangle}
$$

for some $j \in J, 0 \leq k \leq j-1, j \leq N$ and $\zeta \in \Sigma$. Let $\zeta_{m} \in \Sigma$ be such that the first $m$ symbols of $\zeta_{m}$ agree with $\zeta$ but $\sigma^{m}\left(\zeta_{m}\right)=\sigma^{m}(\gamma)$. Then $\left\langle\zeta_{m}\right\rangle-\simeq\langle\gamma\rangle-$, and $\zeta_{m} \rightarrow \zeta$ as $m \rightarrow \infty$. Let $\widehat{d}_{m} \in \mathbb{F} \mathrm{d}\left(\mathbf{X}_{g}\right)$ be such that

$$
\mathrm{Fi}_{g}\left(\widehat{d}_{m}\right)=B_{\left\langle\zeta_{m}\right\rangle-} \cdot Z_{j, N}^{-} W_{j, k}^{N} Z_{j, N}^{+} B_{\left\langle\zeta_{m}\right\rangle} .
$$

Clearly $\widehat{d}_{m} \in K_{\gamma}$ and $\widehat{d}_{m} \rightarrow \widehat{d}$. Thus $K_{\gamma} \cap \mathbb{F} \mathrm{d}\left(\mathbf{X}_{g}\right)$ is a Cantor set.

Lemma 3.8. Let $K_{B_{1}^{\infty}}$ be the composant of $\mathbf{X}_{g}$ such that $K_{B_{1}^{\infty}}$ contains the point $\widehat{r}$ where $\operatorname{Fi}_{g}(\widehat{r})=B_{1}^{-\infty} .1^{\infty}$. Then $K_{B_{1}^{\infty}} \cap \mathbb{F} \mathrm{d}\left(\mathbf{X}_{g}\right)$ is a Cantor set with a countable set of isolated points which have one limit point that is in the Cantor set. 
Proof. Clearly there is a Cantor set in $K_{B_{1}^{\infty}} \cap \mathbb{F} \mathrm{d}\left(\mathbf{X}_{g}\right)$ of points with full itinerary $B_{1}^{\infty} B_{\delta} \cdot B_{\gamma} . B_{1}^{-\infty} . B_{1}^{\infty}$ for some $\delta \in{ }^{N} 2$ and $\gamma \in{ }^{\mathbb{N}} 2$. Suppose that $\widehat{d} \in K_{B_{1}^{\infty}} \cap \mathbb{F} \mathrm{d}\left(\mathbf{X}_{g}\right)$ does not have this form. Then there is an integer $l$ such that $\sigma^{l}\left[\mathrm{Fi}_{g}(\widehat{d})\right]=B_{1}^{-\infty} \cdot 1^{\infty}$, in which case $\widehat{d}$ is isolated in $K_{B_{1}^{\infty}} \cap \mathbb{F} \mathrm{d}\left(\mathbf{X}_{g}\right)$. Notice that the point $\widehat{e}$ with $\operatorname{Fi}_{g}(\widehat{e})=B_{1}^{-\infty} . B_{1}^{\infty}$ is a limit of points with full itinerary $B_{1}^{-\infty} . B_{1}^{K} 1^{\infty}$ for $K \in \mathbb{N}$.

Lemma 3.9. Let $K_{1 \infty}$ be the composant of $\mathbf{X}_{g}$ such that $K_{1 \infty}$ contains the point $\widehat{w}$ with $\mathrm{Fi}_{g}(\widehat{w})=1^{-\infty} .1^{\infty}$. Then $K_{1 \infty} \cap \mathbb{F} \mathrm{d}\left(\mathbf{X}_{g}\right)$ is a countable set.

Proof. Let $\widehat{d} \in K_{1 \infty} \cap \mathbb{F} \mathrm{d}\left(\mathbf{X}_{g}\right)$. Either $\widehat{d}$ has full itinerary $1^{-\infty} \cdot 1^{\infty}$ or there is an integer $l$ such that

$$
\sigma^{l}\left[\mathrm{Fi}_{g}(\widehat{d})\right]=\left\{\begin{array}{l}
1^{-\infty} 0 \cdot 1^{\infty}, \\
1^{-\infty} \cdot B_{1}^{\infty}, \\
1^{-\infty} 01^{k} \cdot B_{1}^{\infty} \quad \text { for some } k \in \mathbb{N} .
\end{array}\right.
$$

Clearly there are only countably many possibilities.

Lemma 3.10. Let $j \in J$ and $0 \leq k \leq j-1$. Let $K_{j, k}$ be the composant of $\mathbf{X}_{g}$ such that $K_{j, k}$ contains the point $\widehat{x}_{j, k}$ with $\mathrm{Fi}_{g}\left(\widehat{x}_{j, k}\right)=W_{j, k}^{-\infty} . W_{j, k}^{\infty}$. Then $K_{j, k} \cap \mathbb{F} \mathrm{d}\left(\mathbf{X}_{g}\right)$ is a countable set of isolated points with a single limit point.

Proof. Let $\widehat{d} \in K_{j, k} \cap \mathbb{F} \mathrm{d}\left(\mathbf{X}_{g}\right)$. Then either $\mathrm{Fi}_{g}(\widehat{d})=W_{j, k}^{-\infty} . W_{j, k}^{\infty}$ or there is an integer $l$ such that $\sigma^{l}\left[\mathrm{Fi}_{g}(\widehat{d})\right]=W_{j, k}^{-\infty} \cdot Z_{j}^{+}$. Clearly this is a countable set and all of the points in the second case are isolated in $K_{j, k} \cap \mathbb{F} \mathrm{d}\left(\mathbf{X}_{g}\right)$ while the first point is a limit of such points.

Lemma 3.11. Let $j \in J$. Let $K_{j}$ be the composant of $\mathbf{X}_{g}$ containing the point $\widehat{z}_{j}$ with $\mathrm{Fi}_{g}\left(\widehat{z}_{j}\right)=Z_{j}^{-} . Z_{j}^{+}$. Then $K_{j} \cap \mathbb{F} \mathrm{d}\left(\mathbf{X}_{g}\right)$ is a Cantor set together with precisely $j$ isolated points.

Proof. First notice that by the choice of $Z_{j}^{-}$, if $\widehat{d} \in \mathbb{F} \mathrm{d}\left(\mathbf{X}_{g}\right)$ with $\mathrm{Fi}_{g}(\widehat{d})=$ $Z_{j}^{-} V_{0} . V_{1}$ and $V_{0} \neq \emptyset$, then $\widehat{d} \notin K_{j}$ as $Z_{j}^{-} \not Z_{j}^{-} V_{0}$ (Lemma 3.1).

Similarly, if there is a negative integer $l$ such that $\sigma^{l}\left[\mathrm{Fi}_{g}(\widehat{d})\right]=Z_{j}^{-} \cdot W_{1}$ then $\widehat{d} \notin K_{j}$. Also if $k \in J$ with $k \neq j$ and $\widehat{d} \in \mathbb{F} \mathrm{d}\left(\mathbf{X}_{g}\right)$ with $\sigma^{l}\left[\operatorname{Fi}_{g}(\widehat{d})\right]=$ $Z_{k}^{-} . W_{1}$ for some word $W_{1}$ in $\{0,1\}$, then $\widehat{d} \notin K_{j}$. Thus the only points $\widehat{d} \in \mathbb{F} d\left(\mathbf{X}_{g}\right)$ that are also in $K_{j}$ have full itinerary

(1) $Z_{j}^{-} \cdot Z_{j}^{+}$,

(2) $Z_{j}^{-} \cdot W_{j, k}^{\infty}$,

(3) $B_{\langle\gamma\rangle-} . B_{\langle\zeta\rangle}$ where $\gamma, \zeta \in \mathbb{N}_{2}$ and $B_{\langle\gamma\rangle-} \simeq Z_{j}^{-}$.

Clearly cases (1) and (3) form a Cantor set of folding points in $K_{j}$. For each $0 \leq k \leq j-1$ let $\widehat{y}_{j, k}$ be in $K_{j} \cap \mathbb{F} \mathrm{d}\left(\mathbf{X}_{g}\right)$ with $\mathrm{Fi}_{g}\left(\widehat{y}_{j, k}\right)=Z_{j}^{-} \cdot W_{j, k}^{\infty}$. 
Notice there are precisely $j$ such points. We will show that each of these points is isolated in $K_{j} \cap \mathbb{F} \mathrm{d}\left(\mathbf{X}_{f}\right)$.

Let $\left(\widehat{w}_{n}\right)_{n \in \mathbb{N}}$ be a sequence of points in $\mathbb{F} \mathrm{d}\left(\mathbf{X}_{g}\right)$ converging to $\widehat{y}_{j, k}$. We will show that there is an integer $N$ such that $\widehat{w}_{n} \notin K_{j}$ for $n \geq N$. The result then follows. Fix $M \in \mathbb{N}$ with $M \geq j^{2}$. Suppose that $\widehat{w}_{n}$ is close enough to $\widehat{y}_{j, k}$ so that the cylinder of $\mathrm{Fi}_{g}\left(w_{n}\right)$ of diameter $M$ agrees with the cylinder of $\mathrm{Fi}_{g}\left(y_{j, k}\right)$ of diameter $M, Z_{j, M}^{-} \cdot W_{j, k}^{M}$. Since $\widehat{w}_{n} \neq \widehat{y}_{j, k}$ there is a largest integer $M^{\prime}$ such that the $M^{\prime}$-cylinder of $\mathrm{Fi}_{g}\left(\widehat{w}_{n}\right)$ ends with the word $W_{j, k}^{M^{\prime}}$. By Lemma 3.6 it must be the case that the cylinder of $\mathrm{Fi}_{g}\left(\widehat{w}_{n}\right)$ of diameter $M^{\prime}$ has the form $Z_{j, M^{\prime}}^{-} . W_{j, k}^{M^{\prime}}$. In fact there must be some $\gamma \in \Sigma$ with

$$
\mathrm{Fi}_{g}\left(\widehat{w}_{n}\right)=B_{\langle\gamma\rangle}-Z_{j, M^{\prime}}^{-} \cdot W_{j, k}^{M^{\prime}} Z_{j, M^{\prime}}^{+} B_{\langle\gamma\rangle} .
$$

By the definition of $\Sigma, B_{\langle\gamma\rangle^{-}} \nsucceq Z_{j}^{-}$. Hence $\widehat{w}_{n}$ is not in $K_{j}$.

Theorem 3.12. Let $\mathcal{K}$ be the collection of composants of $\mathbf{X}_{g}$. Then $\mathcal{K}$ can be partitioned into $\mathcal{K}_{\emptyset}, \mathcal{K}_{c}, \bigcup_{j \in J} \mathcal{K}_{j}$, and $\mathcal{L}$ such that

(1) if $K \in \mathcal{K}_{\emptyset}$ then $K \cap \mathbb{F} \mathrm{d}\left(\mathbf{X}_{g}\right)=\emptyset$;

(2) if $K \in \mathcal{K}_{c}$ then $K \cap \mathbb{F} \mathrm{d}\left(\mathbf{X}_{g}\right)$ is precisely a Cantor set;

(3) if $K \in \mathcal{L}$ then $K \cap \mathbb{F} \mathrm{d}\left(\mathbf{X}_{g}\right)$ is a countable set;

(4) for $j \in J, \mathcal{K}_{j}$ is countable and if $K \in \mathcal{K}_{j}$ then $K \cap \mathbb{F} \mathrm{d}\left(\mathbf{X}_{g}\right)$ is precisely a Cantor set together with $j$ isolated points.

Proof. Let $\mathcal{K}_{\emptyset}$ be the collection of composants of $\mathbf{X}_{g}$ which contain no folding points, and let $\mathcal{K}_{c}$ be the collection of composants described in Lemma 3.7. Let $\mathcal{L}$ be the collection of all composants described in Lemmas 3.8-3.10 together with all of their images under the homeomorphism $\sigma$.

Let $j \in J$ and let $K_{j, 0}$ be the composant $K_{j}$ described in Lemma 3.11. From what was said before we see that $K_{j, 0} \cap \mathbb{F} \mathrm{d}\left(\mathbf{X}_{g}\right)$ is a Cantor set together with precisely $j$ isolated points. For each $m \in \mathbb{Z}$ let $K_{j, m}=\widehat{\sigma}^{m}\left[K_{j, 0}\right]$. Since $\widehat{\sigma}$ is a homeomorphism and $\mathbb{F} d\left(\mathbf{X}_{g}\right)$ is preserved by homeomorphisms, $K_{j, m} \cap \mathbb{F} \mathrm{d}\left(\mathbf{X}_{g}\right)$ is also a Cantor set together with $j$ isolated points. Define $\mathcal{K}_{j}=\left\{K_{j, m}: m \in \mathbb{Z}\right\}$.

Corollary 3.13. There is a family $\mathcal{F}$ of tent map cores such that

(1) $\mathcal{F}$ has cardinality of the continuum, $\mathfrak{c}$

(2) if $f \in \mathcal{F}$ with critical point $c_{f}$ then $\omega\left(c_{f}\right)$ and $\mathbb{F} \mathrm{d}\left(\mathbf{X}_{f}\right)$ are Cantor sets,

(3) if $f \in \mathcal{F}$ then every non-degenerate proper subcontinuum of $\mathbf{X}_{f}$ is an arc,

(4) if $f, g \in \mathcal{F}$ with $f \neq g$ then $\mathbf{X}_{f}$ is not homeomorphic to $\mathbf{X}_{g}$.

Proof. For each $J \subseteq \mathbb{N}$ we can build a map $g_{J}$ as described above from the starting map $f$. This map $g_{J}$ will have the property that $\omega\left(c_{g_{J}}\right)$ and 
$\mathbb{F} \mathrm{d}\left(\mathbf{X}_{g_{J}}\right)$ is a Cantor set but for each $j \in J$ there is a countable collection $\bigcup_{j \in J} \mathcal{K}_{j}$ of composants of $\mathbf{X}_{g_{J}}$ with the property that if $K \in \mathcal{K}_{j}$ then $K \cap$ $\mathbb{F} \mathrm{d}\left(\mathbf{X}_{g_{J}}\right)$ contains $j$ isolated points. For every other composant, $K$, that meets $\mathbb{F} d\left(\mathbf{X}_{g_{J}}\right)$ either $K \cap \mathbb{F d}\left(\mathbf{X}_{g_{J}}\right)$ is a countable set or it is a Cantor set. So if $J, L \subseteq \mathbb{N}$ with $J \neq L$ then $\mathbf{X}_{g_{J}}$ is not homeomorphic to $\mathbf{X}_{g_{L}}$. By our construction the critical point $c_{g}$ is not recurrent so $\mathbf{X}_{g}$ has only arcs as its proper subcontinua (cf. [5]).

For $J \subseteq \mathbb{N}$ let $\mathcal{P}_{J}$ be the set of parameters $r \in(\sqrt{2}, 2]$ of tent map cores for which $\omega\left(c_{f_{r}}\right)$ and $\mathbb{F} \mathrm{d}\left(\mathbf{X}_{f_{r}}\right)$ are both Cantor sets and for which there is a composant $K$ such that $K \cap \mathbb{F} \mathrm{d}\left(\mathbf{X}_{f_{r}}\right)$ contains $j$ isolated points iff $j \in J$. By modifying our initial choice of $f$ in the above construction it is clear that for any $J \subseteq \mathbb{N}, \mathcal{P}_{J}$ is dense in $(\sqrt{2}, 2]$ and has cardinality $\mathfrak{c}$. Also each map with parameter in $\mathcal{P}_{J}$ will have a non-recurrent critical point. This implies that every proper subcontinuum of its inverse limit will be an arc, and also the set of folding points in its inverse limit will be a Cantor set. So in order to distinguish between these inverse limit spaces some new idea is needed.

\section{References}

[1] M. Barge, K. Brucks, and B. Diamond, Self-similarity in inverse limit spaces of the tent family, Proc. Amer. Math. Soc. 124 (1996), 3563-3570.

[2] M. Barge and B. Diamond, Homeomorphisms of inverse limit spaces of one-dimensional maps, Fund. Math. 146 (1995), 171-187.

[3] M. Barge and W. T. Ingram, Inverse limits on $[0,1]$ using logistic bonding maps, Topology Appl. 72 (1996), 159-172.

[4] M. Barge and J. Martin, Endpoints of inverse limit spaces and dynamics, in: Continua (Cincinnati, OH, 1994), Lecture Notes in Pure and Appl. Math. 170, Dekker, New York, 1995, 165-182.

[5] K. Brucks and H. Bruin, Subcontinua of inverse limit spaces of unimodal maps, Fund. Math. 160 (1999), 219-246.

[6] K. Brucks and Z. Buczolich, Trajectory of the turning point is dense for a co- $\sigma$ porous set of tent maps, ibid. 165 (2000), 95-123.

[7] K. M. Brucks and B. Diamond, A symbolic representation of inverse limit spaces for a class of unimodal maps, in: Continua (Cincinnati, OH, 1994), Lecture Notes in Pure and Appl. Math. 170, Dekker, New York, 1995, 207-226.

[8] K. Brucks and M. Misiurewicz, The trajectory of the turning point is dense for almost all tent maps, Ergodic Theory Dynam. Systems 16 (1996), 1173-1183.

[9] H. Bruin, Inverse limit spaces of post-critically finite tent maps, Fund. Math. 165 (2000), 125-138.

[10] P. Collet and J.-P. Eckmann, Iterated Maps on the Interval as Dynamical Systems, Birkhäuser, Boston, 1980.

[11] C. Good, R. Knight, and B. Raines, Non-hyperbolic one-dimensional invariant sets with a countably infinite collection of inhomogeneities, preprint, 2006.

[12] W. T. Ingram, Periodicity and indecomposability, Proc. Amer. Math. Soc. 123 (1995), 1907-1916. 
[13] W. T. Ingram, Inverse Limits, Soc. Mat. Mex., México, 2000.

[14] -, Inverse limits on [0,1] using piecewise linear unimodal bonding maps, Proc. Amer. Math. Soc. 128 (2000), 279-286.

[15] W. T. Ingram and W. S. Mahavier, Interesting dynamics and inverse limits in a family of one-dimensional maps, Amer. Math. Monthly 111 (2004), 198-215.

[16] L. Kailhofer, A partial classification of inverse limit spaces of tent maps with periodic critical points, Topology Appl. 123 (2002), 235-265.

[17] - A classification of inverse limit spaces of tent maps with periodic critical points, Fund. Math. 177 (2003), 95-120.

[18] B. Raines, One-dimensional dynamics and inverse limits, D. Phil. thesis, Oxford Univ., 2002.

[19] - Inhomogeneities in non-hyperbolic one-dimensional invariant sets, Fund. Math. 182 (2004), 241-268.

School of Mathematics and Statistics

University of Birmingham

Birmingham, B15 2TT, UK

E-mail: c.good@bham.ac.uk
Department of Mathematics

Baylor University

Waco, TX 76798-7328, U.S.A.

E-mail: brian_raines@baylor.edu

Received 29 June 2004;

in revised form 8 December 2005 